\title{
A Structured Threshold Model for Mountain Pine Beetle Outbreak
}

\author{
Mark A. Lewis ${ }^{\mathrm{a}, *}$, William Nelson ${ }^{\mathrm{b}}$, Cailin $\mathrm{Xu}^{\mathrm{b}}$ \\ ${ }^{a}$ Department of Mathematical and Statistical Sciences, University of Alberta, Edmonton, \\ Canada \\ ${ }^{b}$ Department of Biological Sciences, University of Alberta, Edmonton, Canada
}

Received: 30 December 2008 / Accepted: 14 September 2009

(C) Society for Mathematical Biology 2009

\begin{abstract}
A vigor-structured model for mountain pine beetle outbreak dynamics within a forest stand is proposed and analyzed. This model explicitly tracks the changing vigor structure in the stand. All model parameters, other than beetle vigor preference, were determined by fitting model components to empirical data. An abrupt threshold for tree mortality to beetle densities allows for model simplification. Based on initial beetle density, model outcomes vary from decimation of the entire stand in a single year, to inability of the beetles to infect any trees. An intermediate outcome involves an initial infestation which subsequently dies out before the entire stand is killed. A model extension is proposed for dynamics of beetle aggregation. This involves a stochastic formulation.
\end{abstract}

Keywords Mountain pine beetle $\cdot$ Structured population model $\cdot$ Integrodifference equation

\section{Introduction}

Mountain pine beetles (Dendroctonus ponderosae Hopkins) are the single most destructive pine forest pest (Logan and Powell, 2001; Logan et al., 2003). Although mountain pine beetles occur naturally throughout the pine forests of western North America, their range is spreading quickly, likely due to changing climate (Logan et al., 2003).

\subsection{Biological background}

Mountain pine beetle outbreaks range from isolated attacks on single trees to 'mass attacks' of virtually all trees in a stand. Mass attacks can decimate forests stands in 1 or 2 years. While the exact determinants of successful outbreaks are unclear, initial beetle attack density and host vigor (as measured by wood production per unit of leaf area) are key factors (Fig. 1). When vigor is low or beetle density high, attacks are successful (tree is killed). When vigor is high or beetle density low, attacks are only partially successful (strip attack) or unsuccessful (tree repels attack).

\footnotetext{
${ }^{*}$ Corresponding author.

E-mail address: mlewis@math.ualberta.ca (Mark A. Lewis).

Published online: 24 October 2009
} 


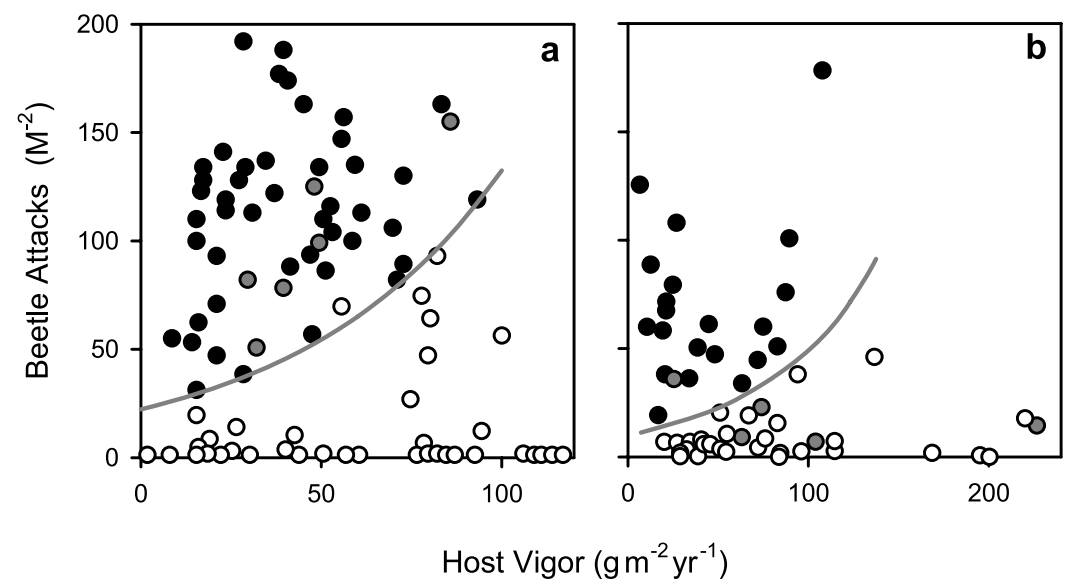

Fig. 1 Empirical thresholds for attack success as a function of host vigor. Each symbol is an individual tree. Solid circles indicate trees were killed by beetles; open circles indicate trees resisted attack and are alive; grey circles indicate trees had a strip attack. The grey lines are empirically estimated thresholds for host mortality. (a) Data for mountain pine beetles attacking lodgepole pine redrawn from Waring and Pitman (1985). The threshold is based the maximum likelihood fit of Eq. (2) with $\beta(v)=\beta_{0} \exp \left(\beta_{1} v\right)$ (see Appendix B for details). (b) Data for spruce bark beetles attacking Norway spruce from Mulock and Christiansen (1986). The axes presented here are converted from the original axes to be comparable with panel (a) (see Appendix A for details). The threshold is redrawn from the original work.

The mountain pine beetle life cycle has been researched intensively, and is well understood. The beetles attack and breed in live host trees-a process that results in host mortality. Most mountain pine beetle populations complete one generation a year (Safranyik and Carroll, 2006) (Fig. 2). Recently developed adults emerge from their host trees in late summer, and take flight in search of new hosts.

The flight period for the entire population is brief, only about 2 weeks long. Dispersal of flying beetles through the forest is guided by two kinds of chemical signals: kairomones, produced by trees, and pheromones, produced by beetles already in the process of attacking new hosts. (See Logan et al., 1998; White and Powell, 1998 for models of these dynamics.)

Given that attack success depends crucially upon tree vigor (Fig. 1), and that beetles can sense tree vigor through kairomones, it is natural to ask whether beetles preferentially attack less vigorous trees over more vigorous. There is some evidence of this so-called primary attraction in mountain pine beetle attacks (Moeck and Simmons, 1991), although is important to note that this is only part of the chemical ecology of mass attack.

Once beetles settle on a potential host, they attempt to bore through the outer bark into the phloem tissue. Healthy trees can resist attacks by producing resin to slow down or stop beetles from constructing tunnels (galleries) to lay eggs. If sufficient beetles attack a particular host, then resin defenses can be overwhelmed, and beetles successfully construct egg galleries in the phloem tissue. The density of beetles required to overcome tree defenses can be measured empirically (Fig. 1). When the attack is successful, the eggs develop into larvae, which create feeding galleries that girdle and often kill the host tree. Beetle populations often over-winter as late instar larvae, and resume development in the spring. Pupation occurs in early spring and adults emerge in late summer. 


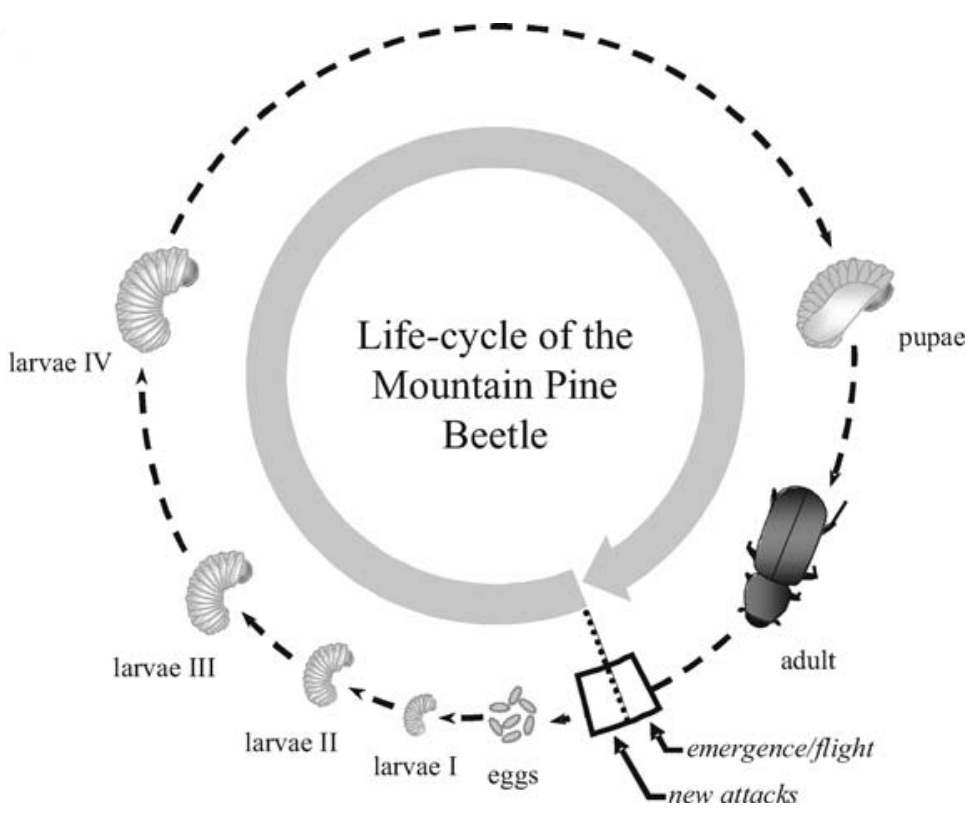

Fig. 2 Life-cycle of the mountain pine beetle. New adults emerge from last season's hosts in late summer, and attack new hosts over a 2-3 week period. If the hosts are killed, then the attacking beetles lay eggs that develop through larval stages over the fall, winter and spring. The major phases of the life-cycle are shown in proportion to their duration over a season. The gray loop indicates 1 year in the model, and the arrow represents the transition point.

\subsection{Models for beetle dynamics}

Models for mountain pine beetle population dynamics differ in their level of ecological detail-ranging from strategic models that phenomenalize the ecological interactions into a single replacement function for the population (Berryman et al., 1984), to complex spatial models that explicitly describe the processes of dispersal, aggregation and attack (Powell et al., 1996). While more complex models are arguably more realistic, it is often difficult to study them and disentangle the contribution of ecological features (White and Powell, 1997). Strategic models, on the other hand, are well suited to study the general dynamics of a system, but often at the expense of realism.

Here, we develop and analyze a strategic population model of the mountain pine beetle to study how host selection and mass attack influence the dynamics of beetle outbreaks. Our work is based on the models of Berryman (1979), Berryman et al. (1984), but provides greater realism by explicitly incorporating how the attack process depends on host vigor.

Early strategic models of mountain pine beetle populations were based on productivity curves for the processes of attack and reproduction at the spatial scale of a forest stand (Berryman, 1979). Productivity curves describe the density of beetles emerging from a stand in a current year, based on the density of attacking beetles in the previous year. While used to depict dynamics at the scale of a forest stand, they are based on observations of per-capita beetle fecundity on individual trees. For aggressive bark beetles, beetle fecundity curves are unimodal, reflecting the balance between overwhelming host 
defenses and reducing reproduction due to overcompensatory competition. For example, if the number of beetles attacking a host is too low, then attacks fail because of host defenses (Fig. 1), but if the number of attacks are too high, then reproduction is reduced because of intraspecific competition. The density of attacking beetles giving rise to the maximum per capita fecundity is the minimum density required to kill the host (Raffa and Berryman, 1983), which implies that hosts with different levels of vigor have different fecundity curves. Thus, the productivity curve for the entire stand, which is the sum of the fecundity curves over all trees, depends on the distribution of vigor in the stand.

Because productivity curve models describe the density of beetles emerging from a stand in a current year based on the density of attacking beetles in the previous year, they offer a straightforward way to predict beetle dynamics. However, they implicitly assume the vigor structure of a stand remains constant through time (Berryman, 1979). Such an assumption is unrealistic for mountain pine beetles because outbreak dynamics alter the forest at a much faster rate than the forest regenerates. A changing vigor structure in a forest implies a dynamic productivity curve. In this paper, we analyze the changing vigor in the forest stand, developing a population model structured by host vigor. We extend the model to include beetle aggregation on the tree hosts.

\subsection{A structured-population model for mass attack}

As demonstrated in Fig. 1, host tree vigor plays a central role in the success or failure of beetle mass attack. Indeed, the beetle density threshold for successful mass attack depends explicitly upon host tree vigor. However, at the level of a forest stand, beetles are likely to encounter variable host tree vigor, as it varies from tree-to-tree. Because the time scale for beetle attack is much faster than the forest regeneration time scale, beetle attack can modify the year-to-year vigor structure in a stand as the attack progresses. If low vigor trees in a stand are attacked early, then the remaining trees may be better able to withstand subsequent attack. In this way, changes in the vigor structure of a stand will affect the year-to-year dynamics of attacking beetle success.

To date, mathematical models for pine beetle attack have generally excluded the vigor structure of the host tree population (but see Raffa and Berryman, 1986 and Powell et al., 1996). However, structured-population models have an established history of revealing key details of processes governing population dynamics (Caswell et al., 1997). A particularly useful outcome of structured-population models is a simplified projection of overall population levels from one year to the next Gurney and Nisbet (1998).

It is the purpose of this paper to investigate the interplay between attack success and primary attraction of beetles to trees, when both of these are affected by the host tree vigor. Specifically, we will investigate conditions on beetle density and tree host vigor required for a successful mass attack. We will use a vigor- and bark area-structured model for attack. The model component will be based on a mixture of an empirical evidence for the attack success, and theoretically-derived relationships for the primary attraction to host trees. As explained in the next section, we will refer to this structured-population model as a "structured-threshold model," as it couples curves of the sort shown in Fig. 1 to beetle production in the subsequent generation.

The model output will include a simplified projection of total beetle population levels from one year to the next as the beetles modify the forest stand structure, destroying trees that remain susceptible to attack at that year's beetle density. We will analyze the 
Table 1 Symbols and variables used the mountain pine beetle production model and its simplification

\begin{tabular}{ll}
\hline Variable & Definition and (units) \\
\hline$t$ & Time index in years $(\mathrm{yr})$ \\
$a$ & Bark area of a tree $\left(\mathrm{m}^{2}\right)$ \\
$v$ & Vigor index of a tree $\left(\mathrm{g} \mathrm{m}^{-2} \mathrm{yr}^{-1}\right)$ \\
$f_{t}(a, v)$ & Distribution of trees, with respect to bark area and vigor of tree \\
& (number per unit area per unit vigor, $\mathrm{yrg}^{-1}$ ) \\
$F_{t}(a, v)$ & Distribution of bark area from all trees with respect to bark area \\
& and vigor of tree (per unit vigor, $\left.\mathrm{g}^{-1} \mathrm{~m}^{2} \mathrm{yr}\right)$ \\
$\chi\left(b_{t}, v\right)$ & Proportion of hosts killed for a given level of host vigor and \\
$b_{t}(a, v)$ & attacking beetle density (dimensionless) \\
$p\left(b_{t}, v\right)$ & Density of attacking beetles (number per unit bark area, $\left.\mathrm{m}^{-2}\right)$ \\
$e_{t}(a, v)$ & Number emerging beetles per attack (dimensionless) \\
$P_{t}\left(b_{t}, a, v\right)$ & Number of emerging beetles from a host tree (dimensionless) \\
$k(a, v)$ & Total number of emerging beetles in the stand (dimensionless) \\
& Preference kernel for trees of given area and vigor \\
$\tilde{k}(v)$ & (per unit vigor per unit area, yr $\mathrm{g}^{-1}$ ) \\
$\phi(v)$ & Preference kernel for trees of given vigor (per unit vigor, $\left.\mathrm{g}^{-1} \mathrm{~m}^{2} \mathrm{yr}\right)$ \\
\hline
\end{tabular}

qualitative dynamics of the simplified projection model, showing how threshold behavior and bistable dynamics in the beetle population levels arise naturally from the underlying structured-population model.

\section{Structured-threshold model}

We develop a general model for host tree and beetle density, structured according to host tree vigor and host tree bark area. We then simplify the model under the assumption that beetles distribute themselves only according to host vigor and not according to host tree bark area. The model variables, their definitions and their units are given in Table 1.

\subsection{Model development}

In our model, we refer to a host as a single tree, and a stand is a group of trees. From the perspective of the mountain pine beetle, host vigor is described by a single index $v$ that characterizes the capacity for resinous defenses. As shown in Fig. 1, an appropriate measure is wood produced per unit leaf area in the host tree. To remain consistent with the empirical evidence, density here refers to the number of beetles per unit area of bark $a$.

The density of attacking beetles on a particular host $i$ is represented by $b\left(a_{i}, v_{i}\right)$, which may vary with both the vigor and size (bark area) of a tree. The number of emerging beetles per attack from host $i$ is given by the function $p\left(b\left(a_{i}, v_{i}\right), v_{i}\right)$, which depends on both attack density and host vigor. This function includes the probability of host mortality, as well as the effects of intraspecific competition. In the early literature, it is referred to as the "productivity curve." Empirical evidence suggests that the productivity curve is a threshold function over $b\left(a_{i}, v_{i}\right)$ at any particular value of $v$. For example, we could use

$$
p(b(a, v), v)=\chi(b(a, v), v) A e^{-\gamma b(a, v)}
$$




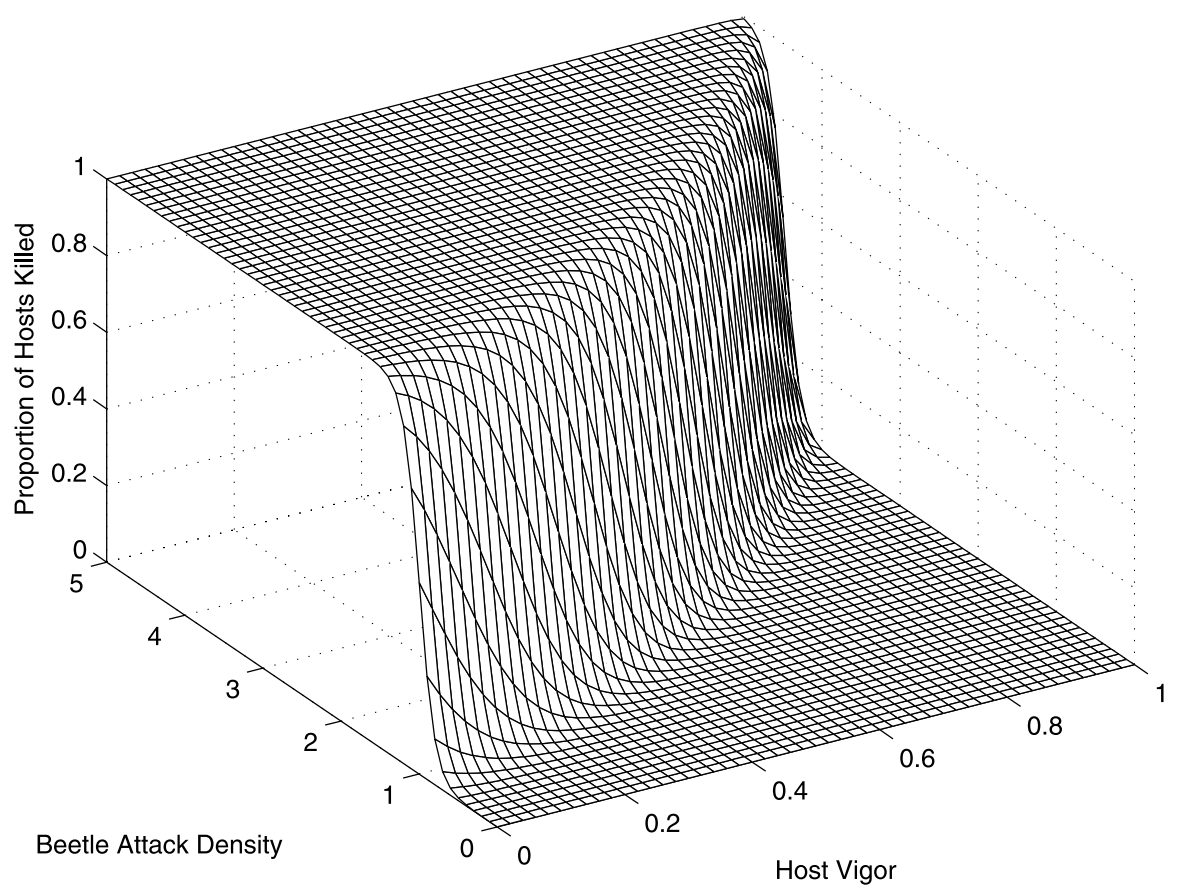

Fig. 3 The function $\chi(b(v), v)$, which is the proportion of trees killed in vigor class $v$ by attacking beetle density $b(v)$. The dimensionless form (1)-(2) with the exponential function $\left(\beta(v)=\exp \left(\beta_{1} v\right)\right)$ is shown here for $\alpha=10$ and $\beta_{1}=1.5$.

where

$$
\chi(b(a, v), v)=\left(1+e^{-\alpha(b(a, v)-\beta(v))}\right)^{-1} .
$$

The function $\chi(b(a, v), v)$ represents the proportion of hosts killed for a given level of host vigor and attacking beetle density, and $\beta(v)$ defines the line where $\chi(b(a, v), v)=$ 0.5 (Fig. 3). The maximum likelihood fit of $\beta(v)=\beta_{0} \exp \left(\beta_{1}(v)\right)$ to attack data is shown in Fig. 1. This curve gives a slightly better fit than the original linear function of Waring and Pitman (1985).

The parameter $\alpha$ and the function $\beta(v)$ describe the expected probability of host mortality $\chi$. Empirical evidence suggests that the transition from low to high probability of mortality is rapid, and that the function $\beta(v)$ is a monotonically increasing function of host vigor (Fig. 1).

The parameters $\gamma$ and $A$ reflect the effects of intraspecific competition in the beetles. Researchers have found that the influence of competition begins at attack densities greater than the attack threshold, and that the strength of competition is independent of host vigor (e.g., Raffa and Berryman, 1983). This suggests that the processes of host defense and intraspecific competition can be modeled independently as shown in (1) (Berryman et al., 1984). Equation (1) gives a non-monotonic growth function, with the possibility of 
Table 2 Parameters used in the mountain pine beetle model. Details of calculation of the parameters are given in Appendix B

\begin{tabular}{lll}
\hline Parameter & Estimate (dimensionless) & Source \\
\hline$\alpha$ threshold steepness & $0.21 \mathrm{~m}^{2}(4.69)$ & Waring and Pitman (1985) \\
$A$ beetle growth & $32.9(32.9)$ & Raffa and Berryman (1983) \\
$S$ dispersal survival & Variable & - \\
$\beta_{0}$ attack threshold & $22.34 \mathrm{~m}^{-2}(1)$ & Waring and Pitman (1985) \\
$\beta_{1}$ nonlinear threshold & $0.0178 \mathrm{~g}^{-1} \mathrm{~m}^{2} \mathrm{yr}^{1}(2.1)$ & Waring and Pitman $(1985)$ \\
$\gamma$ density dependence & $0.0166 \mathrm{~m}^{-2}(0.371)$ & Raffa and Berryman $(1983)$ \\
$v_{m}$ maximum vigor & $116.4 \mathrm{~g} \mathrm{~m}^{-2} \mathrm{yr}^{-1}(1)$ & Waring and Pitman $(1985)$ \\
$A_{T}$ average bark area & $12.6 \mathrm{~m}^{2}(-)$ & He (2006) \\
\hline
\end{tabular}

overcompensation in the beetle dynamics. In this paper, we will estimate $\gamma$ and $A$ from the data in Raffa and Berryman (1983) (Table 2, Appendix B). is

The total number of beetles that emerge from a given host tree with area $a$ and vigor $v$

$$
e(a, v)=\underbrace{p\left(b\left(a, v_{i}\right), v\right)}_{\text {beetles per attack }} \cdot \underbrace{a}_{\text {bark area }} \cdot \underbrace{b(a, v)}_{\text {attackers per unit area bark }} .
$$

We define the distribution of host structure as $f(a, v)$, The total number of trees in the stand is

$$
N=\int_{0}^{\infty} \int_{0}^{\infty} f(a, v) d a d v
$$

and $f(a, v) / N$ is a probability density function for the joint distribution of tree area and vigor.

The total number of emerging beetles over the entire stand in any given year $t$ is $e(a, v)$ weighted by $f(a, v)$

$$
\begin{aligned}
P_{t} & =\int_{0}^{\infty} \int_{0}^{\infty} \underbrace{f_{t}(a, v)}_{\text {host type distribution }} \cdot \underbrace{a p\left(b_{t}(a, v), v\right) b_{t}(a, v)}_{\text {number emerging from host }} d a d v \\
& =\int_{0}^{\infty} \int_{0}^{\infty} \underbrace{F_{t}(a, v)}_{\text {bark area density }} \cdot \underbrace{p\left(b_{t}(a, v), v\right) b_{t}(a, v)}_{\text {density emerging from host }} d a d v
\end{aligned}
$$

where $F_{t}(a, v)=a f_{t}(a, v)$ is the distribution of total bark area with respect to the bark area of a tree and vigor of a tree.

If we assume that the forest dynamics of growth, competition, and non-beetle caused mortality are very slow relative to the change caused by beetle attacks, then the distribution of potential hosts is given by

$$
f_{t+1}(a, v)=f_{t}(a, v)\left(1-\chi\left(b_{t}(a, v), v\right)\right) .
$$

In effect, we are modeling an epidemic moving through an otherwise static forest. Multiplying Eq. (6) by bark area $a$ yields

$$
F_{t+1}(a, v)=F_{t}(a, v)\left(1-\chi\left(b_{t}(a, v), v\right)\right) .
$$


Attack densities and distribution of host structure will change from year to year. The balance equation for attack densities is the number of emerging beetles redistributed over the new hosts in the stand.

$$
\begin{aligned}
b_{t+1}(a, v) & =K_{t+1}(a, v) P_{t} \\
& =K_{t+1}(a, v) \int_{0}^{\infty} \int_{0}^{\infty} F_{t}(a, v) p\left(b_{t}(a, v), v\right) b_{t}(a, v) d a d v
\end{aligned}
$$

where $K_{t+1}(a, v)$ is a function that redistributes the attacking beetles onto the available bark area that is left after infestation. The hosts will have already suffered damage from the beetle infestation at time $t$, and so the available bark area is $F_{t+1}(a, v)$.

It is assumed that proportion $S$ of the beetles survive the redistribution process. Hence, the function $K_{t+1}(a, v)$ must conserve the number of attacking beetles so that the density of attacking beetles, weighted by the total available bark area equals the total number of emerging beetles

$$
\int_{0}^{\infty} \int_{0}^{\infty} b_{t+1}(a, v) F_{t+1}(a, v) d a d v=S P_{t} .
$$

Multiplying both sides of (8) by $F_{t+1}(a, v)$, integrating, and applying Eq. (9) yields

$$
\int_{0}^{\infty} \int_{0}^{\infty} K_{t+1}(a, v) F_{t+1}(a, v) d a d v=S .
$$

If the beetles have no preference regarding tree bark area or vigor then the choice

$$
K_{t+1}(a, v)=\frac{S}{\int_{0}^{\infty} \int_{0}^{\infty} F_{t+1}(a, v) d a d v}
$$

will distribute the beetles evenly with respect to these factors. In this case $K_{t+1}$ is a constant in Eq. (8), and hence the density of beetles per unit area bark $b_{t+1}$ is a constant. Note that the actual distribution of beetles, given by $b_{t+1} F_{t+1}(a, v)$, is nonconstant, and is equal to zero for those bark area and vigor values where there are no trees $\left(f_{t+1}(a, v)=0\right)$.

Alternatively, we can define a preference function $k(a, v)$, with preference function values larger than one indicating more preferred tree bark area and vigor, and preference function values less than one indicating less preferred tree bark area and vigor. The modification of Eq. (11) that includes the preference function is

$$
K_{t+1}(a, v)=\frac{S k(a, v)}{\int_{0}^{\infty} \int_{0}^{\infty} k(a, v) F_{t+1}(a, v) d a d v} .
$$

Here, the preference function $k(a, v)$ appears both in the numerator and under the integral sign in the normalization constant in the denominator. Equations (8) and (12) become

$$
b_{t+1}(a, v)=k(a, v) \frac{\int_{0}^{\infty} \int_{0}^{\infty} F_{t}(a, v) \chi\left(b_{t}(a, v), v\right) b_{t}(a, v) S A e^{-\gamma b(a, v)} d a d v}{\int_{0}^{\infty} \int_{0}^{\infty} k(a, v) F_{t}(a, v)\left(1-\chi\left(b_{t}(a, v), v\right)\right) d a d v}
$$


The model system of equations, (1)-(2), (7) and (13), is a nonlinear integrodifference system. The unknown functions to be solved for are the density of attacking beetles $b_{t}(a, v)$ and the distribution of total bark area not yet destroyed by infestation $F_{t}(a, v)$.

In reality, we expect that beetle aggregation as well as tree preference will play a role in the distribution of beetles. We defer incorporation of this beetle aggregation to Sect. 4 .

\subsection{Model simplification}

Although beetles can respond to size cues for trees (Cole and McGregor, 1983), we focus on the minimal conditions under which outbreak can occur and assume that attacking beetles distribute themselves only according to host vigor, independently of tree size, and are able to attack the lower vigor trees preferentially. Although this undoubtedly an oversimplification of dynamics of tree preference by beetles, it can be considered a "bestcase" establishment scenario, where by choosing low-vigor trees, beetles can establish most easily. Then the above system can be simplified. Define $\phi(v)$ as the distribution of bark area of live hosts as a function of host vigor, so

$$
\phi_{t}(v)=\int_{0}^{\infty} F_{t}(a, v) d a
$$

The total bark area of live hosts per hectare in the stand is given by

$$
M_{t}=\int_{0}^{\infty} \phi_{t}(v) d \nu
$$

If we assume that emerging beetles only redistribute themselves by host vigor, then the redistribution function $K_{t+1}$ depends only upon host vigor $v$. Substitution into Eq. (13) shows that $b_{t+1}$ depends only upon host vigor $v$. Therefore, Eq. (13) becomes

$$
b_{t+1}(v)=\tilde{k}(v) \frac{\left(\int_{0}^{\infty} \phi_{t}(v) \chi\left(b_{t}(v), v\right) b_{t}(v) A e^{-\gamma b_{t}(v)} d v\right)}{\int_{0}^{\infty} \tilde{k}(v) \phi_{t}(v)\left(1-\chi\left(b_{t}(v), v\right)\right) d v} .
$$

Integration of (7) yields

$$
\phi_{t+1}(v)=\phi_{t}(v)\left(1-\chi\left(b_{t}(v), v\right)\right) \text {. }
$$

We assume that the maximum vigor level possible is $v_{m}$, and that the average level of bark area initially available is $\bar{\phi}_{0}$. The threshold location function $\beta(v)$ is assumed to have the form $\beta(v)=\beta_{0} \exp \left(\beta_{1} v\right)$. While it would be possible to use the original Waring and Pitman (1985) straight-line function $\beta(v)=\beta_{0}+\beta_{1} v$, this function does not fit the data as well (Fig. 1), and is no simpler in the subsequent analysis. The initial number of beetles is given by

$$
N_{0}=\int_{0}^{v_{m}} \phi_{0}(v) b_{0}(v) d v .
$$




\subsection{Nondimensionalization}

To facilitate analysis, we rescale the variables to be dimensionless

$$
v^{*}=\frac{v}{v_{m}}, \quad b^{*}=\frac{b}{\beta_{0}}, \quad \phi^{*}=\frac{\phi}{\bar{\phi}_{0}} .
$$

Corresponding dimensionless parameters are given by

$$
\gamma^{*}=\gamma \beta_{0}, \quad \alpha^{*}=\alpha \beta_{0}, \quad \beta_{1}^{*}=\beta_{1} v_{m}, \quad N_{0}^{*}=\frac{N_{0}}{v_{m} \beta_{0} \bar{\phi}_{0}}, \quad A^{*}=S A
$$

and functions by

$$
\tilde{k}^{*}\left(v^{*}\right)=v_{m} \tilde{k}\left(v^{*} v_{m}\right), \quad \chi^{*}\left(b^{*}\left(v^{*}\right), v^{*}\right)=\left(1+e^{-\alpha^{*}\left(b^{*}-\left(\exp \left(\beta_{1}^{*} v^{*}\right)\right)\right)}\right)^{-1} .
$$

Dropping asterisks for notational simplicity, we rewrite Eq. (16) in its dimensionless form

$$
b_{t+1}(v)=\tilde{k}(v) \frac{\left(\int_{0}^{1} \phi_{t}(v) \chi\left(b_{t}(v), v\right) b_{t}(v) A e^{-\gamma b_{t}(v)} d v\right)}{\int_{0}^{1} \tilde{k}(v) \phi_{t}(v)\left(1-\chi\left(b_{t}(v), v\right)\right) d v},
$$

with $0<v<1$ and the average initial bark density equal to one $\bar{\phi}_{0}=1$. Equation (17) remains unchanged.

\section{A population projection model}

One mathematical approach for simplifying a structured-population model to a simplified, nonstructured population model is to use a method of population projection (Powell et al., 1996) This entails assuming a particular form structured variable (in this case tree host vigor) which then allows for simplification. In our case, we restrict ourselves to the case where the distribution of vigor of the trees is initially uniform (i.e., each possible vigor level $0<v<1$ is equally likely, so that $\phi_{0}=1$ ). By way of example, we consider the case of an exponential preference function, with attraction to the lower vigor trees $\tilde{k}(v)=c \exp (-c v)$.

\subsection{A recurrence relation for beetle density at low host vigor}

In this section, we derive a recurrence relation for beetle density at low host vigor $\tilde{b}_{t}$. We assume that the beetles are initially distributed according to the preference function $\tilde{k}(v)$ so that

$$
b_{0}(v)=\tilde{b}_{0} \exp (-c v)
$$

where

$$
\tilde{b}_{0}=N_{0} \frac{c}{1-\exp (-c)}
$$




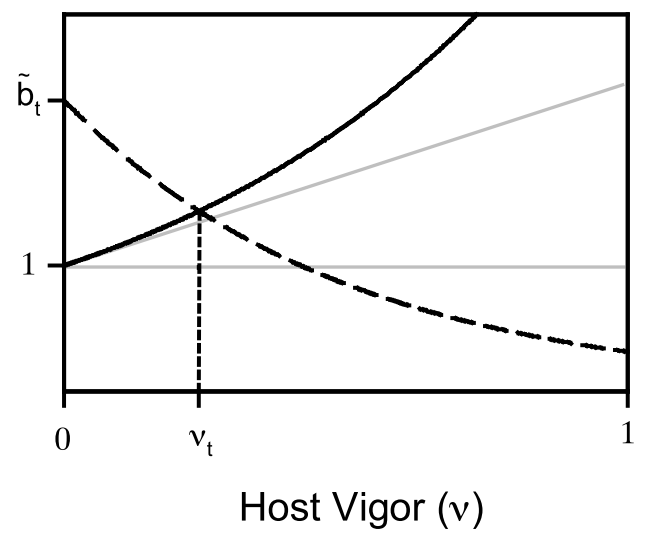

Fig. 4 Host preference and attack success threshold. The attack success threshold $\beta(v)$ (solid line indicating location of rapid transition in Fig. 3) increases with increasing host vigor, reflecting that hosts with higher vigor require more beetles to be successfully attacked. Host preference (dashed line) decreases with host vigor, reflecting the behavioral preference that beetles show for low vigor trees. The vigor level $\left(v_{t}\right)$ where these two curves intersect (dotted line) is the maximum vigor level where attacking beetles in year $t$ will successfully overwhelm host defenses. Beetles that attack hosts with vigor levels greater than $v_{t}$ are killed without reproducing. The dark solid curve shows the exponential attack threshold $\beta(v)=\exp \left(\beta_{1} v\right)$ with $\beta_{1}=1.5$. The light solid curves show the linear attack threshold $\beta(v)=1+\beta v$, with $v>0$ and $v=0$. The dashed curve shows the host preference with $c=2$, and $N=1$.

is chosen to ensure that $\int_{0}^{1} b_{0}(v) d v=N_{0}$. Here, $\tilde{b}_{0}$ can be interpreted as the beetle density for low host vigor. To simplify the analysis, we consider the case where $\alpha \rightarrow \infty$ so that $\chi \rightarrow H\left(b(v)-\exp \left(\beta_{1} v\right)\right)$ where $H$ is the Heaviside step function. In other words, for a given vigor level $v$, the beetles are unsuccessful $(\chi(b(v), v)=0)$ if the beetle density is subthreshold $\left(b(v)<\exp \left(\beta_{1} v\right)\right)$, and are successful $(\chi(b(v), v)=1)$ if the beetle density is superthreshold $\left(b(v)>\exp \left(\beta_{1} v\right)\right)$. Beetle success results in reproduction, and also in the killing of the host.

Under these assumptions, the condition for the beetle population to initially reproduce is $b_{0}(v)-\exp \left(\beta_{1} v\right)>0$ for some value or $v$. The value of $v$ which gives the largest lefthand side is $v \rightarrow 0$, and hence the condition for initial beetle reproduction is $\tilde{b}_{0}>1$. This is satisfied if either enough beetles are introduced ( $N_{0}$ sufficiently large), or the preference for low vigor trees is pronounced ( $c$ sufficiently large).

If initial number of beetles introduced $N_{0}$ is very large, then the beetle population may successfully reproduce for all vigor levels. This occurs when $b(v)-\exp \left(\beta_{1} v\right)>0$ for all $v$. The value of $v$ that gives the smallest left-hand side is $v=1$. Hence, the condition for successful reproduction for all vigor levels becomes $b_{0}(1)>\exp \left(\beta_{1}\right)$, or equivalently, $\tilde{b}_{0}>\left(\exp \left(\beta_{1}\right)\right) \exp (c)$. When this condition is satisfied, the entire stand is infested with beetles and destroyed in a single time step.

At intermediate values of $N_{0}$ the low vigor trees will host beetles, and the high vigor trees will not (Fig. 4). In this case, there is threshold tree vigor level $v_{0}$, below which the beetle reproduces (and also kills the trees), and above which the beetle does not reproduce, and the trees are left unscathed. The threshold vigor value $v_{0}$ is calculated as the value of $v$ satisfying $b_{0}(v)=\exp \left(\beta_{1} v\right)$. Using Eq. (23), this is rewritten as $\tilde{b}_{0} \exp (-c v)=\exp \left(\beta_{1} v\right)$. 
This can be solved explicitly

$$
v_{0}=\frac{1}{c+\beta_{1}} \log \left(\tilde{b}_{0}\right) .
$$

Mathematically, the threshold function $\chi=1$ for $0<v<v_{0}$ and $\chi=0$ for $v_{0}<v<1$. Using this idea, we can rewrite (22) for $t=0$ as

$$
b_{1}(v)=\tilde{b}_{1} \exp (-c v),
$$

where

$$
\tilde{b}_{1}=\frac{\int_{0}^{\nu_{0}} \tilde{b}_{0} c \exp (-c v) A e^{-\gamma \tilde{b}_{0} \exp (-c v)} d v}{\int_{v_{0}}^{1} c \exp (-c \nu) d v} .
$$

Integrating (26) with respect to $0<v<1$ yields the number of beetles in the stand after one time step as $N_{1}=\tilde{b}_{1}(1-\exp (-c)) / c$.

If we assume that the beetle population has grown and spread through increasing vigor classes over successive generations, then $\phi_{t+1}(v)$ is zero for $0<v<v_{t+1}$ and is one for $v_{t+1}<v<1$. Here, $v_{t+1}$ satisfies $\tilde{b}_{t+1} \exp (-c v)=\exp \left(\beta_{1} v\right)$, and hence

$$
v_{t+1}=\frac{1}{c+\beta_{1}} \log \left(\tilde{b}_{t+1}\right),
$$

where

$$
b_{t+1}(v)=\tilde{b}_{t+1} \exp (-c v),
$$

and

$$
\tilde{b}_{t+1}=\frac{\int_{v_{t-1}}^{v_{t}} \tilde{b}_{t} c \exp (-c v) A e^{-\gamma \tilde{b}_{t} \exp (-c v)} d v}{\int_{v_{t}}^{1} c \exp (-c v) d v} .
$$

This expression yields a positive $\tilde{b}_{t+1}$ providing $v_{t}>v_{t-1}$. Thus, Eqs. (28) and (30) are valid so long as $v_{t}$ is an increasing sequence with values below 1 . This generates a corresponding, increasing sequence of $\tilde{b}_{t}$ values. When $v_{t} \leq v_{t-1}$, the population dies. When $v_{t} \geq 1$ the entire stand has been invaded.

Equation (30) also satisfies Eq. (27) for the case $t=0$. If the substitution $\tilde{b}_{-1}=1$ is made, then Eq. (28) yields $v_{-1}=\left(c+\beta_{1}\right)^{-1} \log \left(\tilde{b}_{-1}\right)=0$, indicating that prior to the infestation outbreak there are no infected trees. As above, $\tilde{b}_{t}$ can be translated into the total number of beetles in the stand $N_{t}$ by integrating (29) from $v_{t}$ to 1 to yield

$$
N_{t}=\frac{\tilde{b}_{t}\left(\tilde{b}_{t}^{-c /\left(c+\beta_{1}\right)}-e^{-c}\right)}{c} .
$$

Perhaps surprisingly, Eq. (30) can be integrated exactly. However, the cases with and without density-dependence ( $\gamma$ greater than zero and equal to zero) must be treated separately. In the next section, we analyze the density-independent case $(\gamma=0)$. In the following section, we analyze the density-dependent case $(\gamma>0)$. 


\subsection{Linear growth model}

When $\gamma=0$, integration of Eq. (30) yields

$$
\tilde{b}_{t+1}=A \frac{\tilde{b}_{t}\left(\exp \left(-c v_{t-1}\right)-\exp \left(-c v_{t}\right)\right)}{\exp \left(-c v_{t}\right)-\exp (-c)} .
$$

Equation (28) allows us to rewrite the right-hand side in terms of $\tilde{b}_{t}$ and $\tilde{b}_{t-1}$

$$
\tilde{b}_{t+1}=A \frac{\tilde{b}_{t}\left(\tilde{b}_{t}^{\frac{\beta_{1}+c}{c}}-\tilde{b}_{t-1}^{\frac{\beta_{1}+c}{c}}\right)}{\tilde{b}_{t-1}^{\frac{\beta_{1}+c}{c}}\left(1-\tilde{b}_{t}^{\frac{\beta_{1}+c}{c}} \exp (-c)\right)} .
$$

Equation (33) is a discrete-time dynamical system that describes the progression of disease through a stand structured according to vigor. Starting with the initial condition $\tilde{b}_{-1}=1$ and $\tilde{b}_{0}$ given by Eq. (24), we can evaluate $\tilde{b}_{t+1}$ for successive time steps, calculate the corresponding spread through the vigor classes from Eq. (28) and the corresponding total beetle numbers from (31).

What are the possible outcomes of such a calculation? If the beetle population is reproducing, it must be above threshold $(\chi(b(v), v)=1)$ in a region where bark density $\phi(v)$ is nonzero. Because the beetle destroys all available trees at all vigor levels where it is above threshold, and this is not replaced, a reproducing population has $\tilde{b}_{t+1}>\tilde{b}_{t}$. In other words, in each time a reproducing beetle population invades and destroys higher vigor classes. Hence, if the sequence of $\tilde{b}_{t}$ values from Eq. (33) starts to decline, then the beetle population has gone extinct. This can happen, even when the growth rate $A$ is much larger than unity, because the beetle is destroying its resource, the forest bark, as time progresses.

This excludes the possibility of an endemic population. However, an endemic population may be possible in a more complex model where trees change vigor classes as they mature. This is a subject for further research.

If the beetle population does not die out before the entire stand is consumed, the alternative is that the $\tilde{b}_{t}$ values grows monotonically past the critical value $\tilde{b}_{t}=\exp \left(c+\beta_{1}\right)$. At this point, the entire structured stand (as described by all vigor classes of size less than or equal to the maximum value, one) is destroyed.

\subsection{Nonlinear growth model}

Integration of (30) for the case $\gamma>0$ yields

$$
\tilde{b}_{t+1}=\frac{A}{\gamma} \frac{e^{-\gamma \tilde{b}_{t} \exp \left(-c v_{t}\right)}-e^{-\gamma \tilde{b}_{t} \exp \left(-c v_{t-1}\right)}}{\exp \left(-c v_{t}\right)-\exp (-c)} .
$$

Note that, even though this is undefined for the linear model $(\gamma=0)$, it converges to the solution of the linear model (32) as $\gamma \rightarrow 0$. This calculation can be facilitated by Taylor expansion of the exponential terms in the numerator to leading order in $\gamma$. Equation (28) allows us to rewrite the right-hand side in terms of $\tilde{b}_{t}$ and $\tilde{b}_{t-1}$

$$
\tilde{b}_{t+1}=\frac{A \tilde{b}_{t}^{\frac{\beta_{1}+c}{c}}}{\gamma} \frac{\exp \left(-\gamma \tilde{b}_{t} \tilde{b}_{t}^{-\frac{\beta_{1}+c}{c}}\right)-\exp \left(-\gamma \tilde{b}_{t} \tilde{b}_{t-1}^{-\frac{\beta_{1}+c}{c}}\right)}{1-\tilde{b}_{t}^{\frac{\beta_{1}+c}{c}} \exp (-c)} .
$$



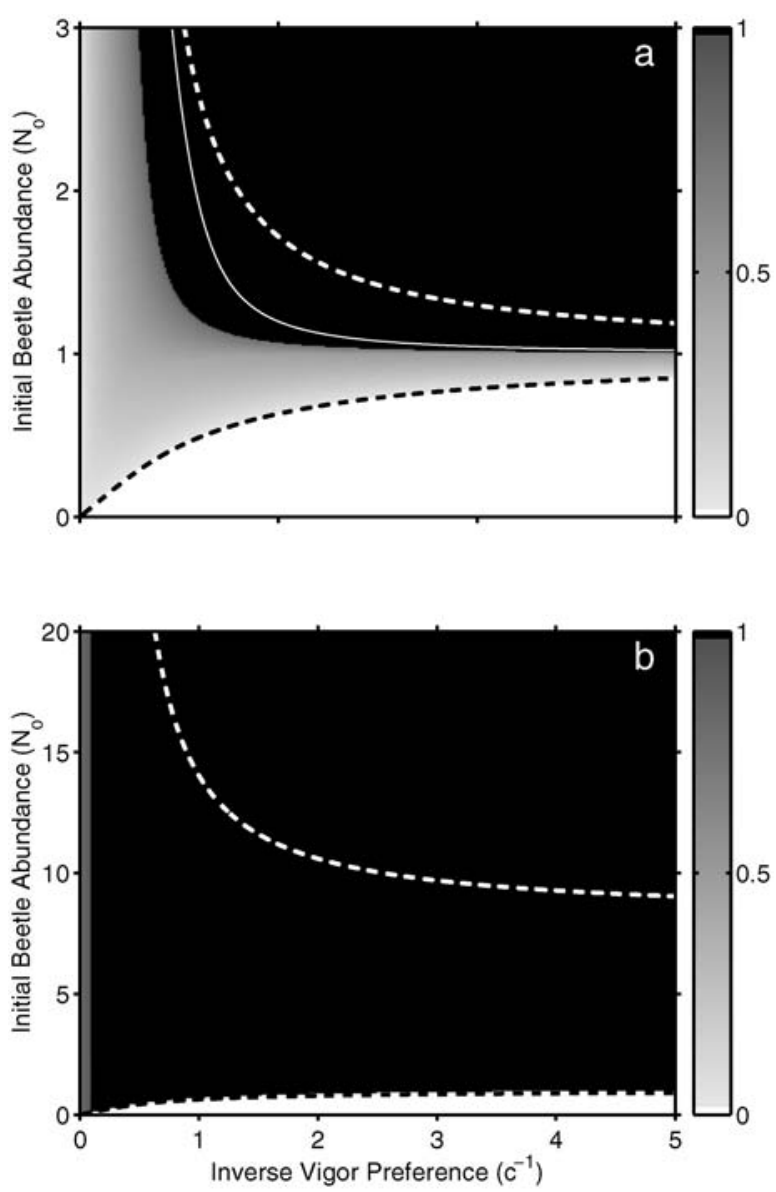

Fig. 5 Forest damage as a function of initial beetle density and host preference predicted by the structured threshold model. The cumulative proportion of trees killed at the end of an infestation is shown by the gray-scale, where white represents zero host mortality and black represents the mortality of all trees in the stand (color bar). The dashed black line depicts the minimum beetle density required to successfully kill the weakest hosts, and the dashed white line depicts the density above which all hosts are killed in the first year of the infestation. The solid white line in panel (a) shows the initial beetle numbers guaranteed to eventually kill the entire stand, calculated from Eq. (40). Axes values that are above and to the right of the solid line give rise to populations that grow in the second year. (a) Dynamics with a constant attack threshold $(A=2$, $\gamma=0.8$, and $\beta=0$ ). (b) Dynamics using empirically derived parameters $(A=33, \gamma=0.37$, and $\beta=2.1)$. Initial beetle abundance can be translated back to dimensional units using Eq. (20).

As before, we have a discrete-time dynamical system with initial condition $\tilde{b}_{-1}=1$ and $\tilde{b}_{0}$ given by Eq. (24), whose solution $\tilde{b}_{t+1}$, calculated for successive time steps, yields the extent of the spread through vigor classes from Eq. (28) and the corresponding total beetle numbers from (31). 


\subsubsection{A lower bound for mass attack shows threshold effects}

To demonstrate a threshold effect, we consider a simplified system where the attack success threshold is constant ( $\beta_{1}=0$; see Fig. 4). We can rewrite Eqs. (33) and (35) in terms of the ratio $r_{t}=\tilde{b}_{t} / \tilde{b}_{t-1}$ to give

$$
r_{t+1}=A \frac{r_{t}-1}{1-\tilde{b}_{t} \exp (-c)}=f\left(r_{t}, \tilde{b}_{t}\right)
$$

and

$$
r_{t+1}=\frac{A}{\gamma} \frac{\exp (-\gamma)-\exp \left(-\gamma r_{t}\right)}{1-\tilde{b}_{t} \exp (-c)}=g\left(r_{t}, \tilde{b}_{t}\right)
$$

The initial conditions to these equations are given by $r_{0}=\tilde{b}_{0} / \tilde{b}_{-1}=\tilde{b}_{0}>1$ (Eq. (24)).

We consider an approximations to the dynamical Eqs. (36) and (37) which provides a lower bound $\underline{r}_{t}$ for $r_{t}$. The approximation is

$$
\underline{r}_{t+1}=f\left(\underline{r}_{t}, \underline{r}_{t}\right)=A \frac{\underline{r}_{t}-1}{1-\underline{r}_{t} \exp (-c)}
$$

for Eq. (36) and

$$
\underline{r}_{t+1}=g\left(\underline{r}_{t}, \underline{r}_{t}\right)=\frac{A}{\gamma} \frac{\exp (-\gamma)-\exp \left(-\gamma \underline{r}_{t}\right)}{1-\underline{r}_{t} \exp (-c)}
$$

for Eq. (37). To show that $\underline{r}_{t}$ is a lower bound for $r_{t}$ observe that $f$ and $g$ are increasing functions of their second argument $\tilde{b}_{t}$. In turn, $\tilde{b}_{t}=r_{t} r_{t-1} \cdots r_{1} r_{0}$ is bounded below by $r_{t}$ because the increasing sequence $\tilde{b}_{s}$ implies $r_{s}=\tilde{b}_{s} / \tilde{b}_{s-1}>1$ for $0<s<t-1$.

The equations can be analyzed graphically using cobwebbing (Fig. 7). For each system, there is a unique positive unstable steady state $r^{*}$ which is larger than one. For Eq. (38), the steady state is $\underline{r}^{*}=\left(\sqrt{(A-1)^{2}+4 \exp (-c)}-(A-1) /(2 \exp (-c))\right.$. For Eq. (39), the steady state must be found numerically. Providing $\underline{r}_{0}>r^{*}$, the cobwebbing shows $\underline{r}_{t}>\underline{r}^{*}$ for all $t$. This also holds true for $\tilde{b}_{0}$ : if $\tilde{b}_{0}$ exceeds $\underline{r}^{*}$ it also will remain larger than $r^{*}$ for all $t$. This is because $\tilde{b}_{0}=r_{0}$ and $\underline{r}_{t}$ is a lower bound for $r_{t}$. The implication is that when $\tilde{b}_{0}$ exceeds $\underline{r}^{*}$ the beetle will eventually destroy the entire stand. Employing (24) to translate this to a constraint on $N_{0}$ yields a critical number beetles required to eventually destroy the entire stand as

$$
N_{0 c}=r^{*} \frac{1-\exp (-c)}{c} .
$$

This is an upper bound for the number of beetles required to destroy the entire stand. As can be seen in Fig. 5(a), fewer beetles may also suffice to destroy the entire stand.

When $\underline{r}_{0}$ lies between 1 and $\underline{r}^{*}$, the ratio $\underline{r}_{t}$ declines to an $\underline{r}_{t}$ value below one. The threshold for declining $r_{t}$ lies below $r^{*}$ because $\underline{r}_{t}$ is a lower bound for $r_{t}$. This threshold is calculated numerically in Fig. 5(a) for the linear model. 

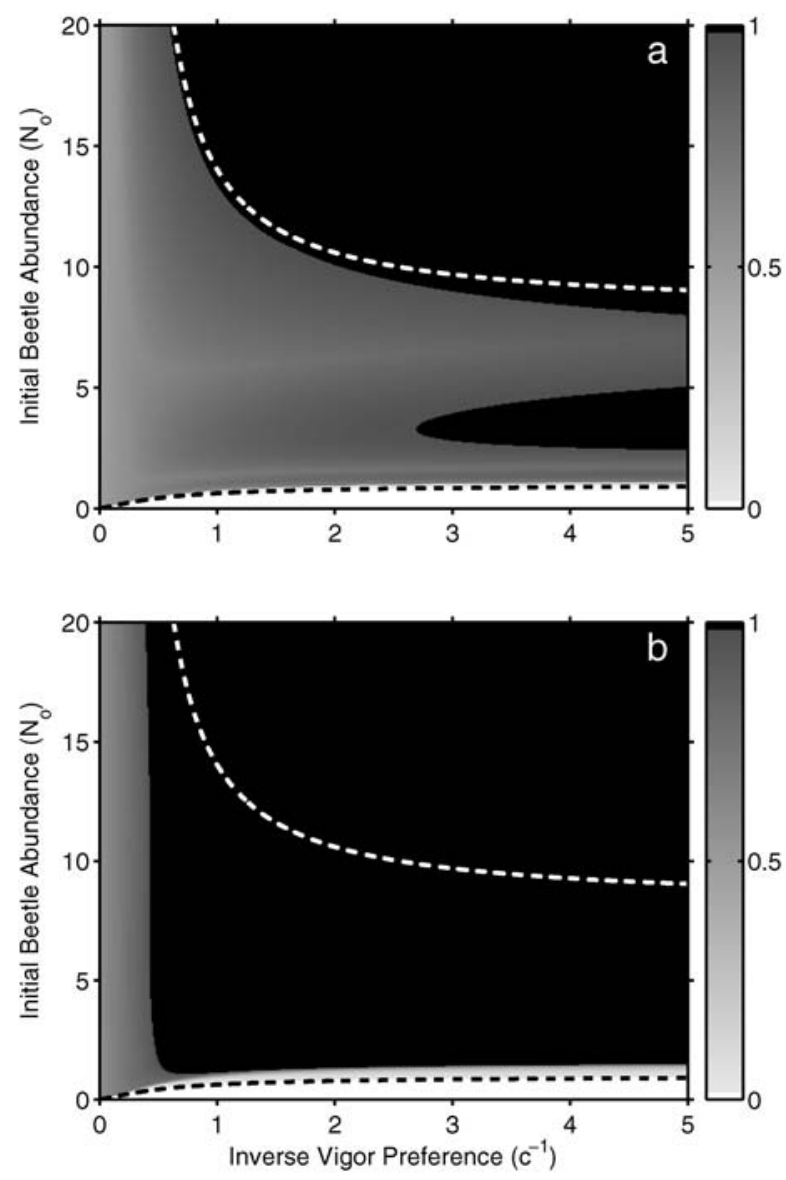

Fig. 6 Forest damage as a function of initial beetle density and host preference predicted by the structured threshold model. See Fig. 5 for legend details. (a) Dynamics with increased intraspecific competition ( $A=33, \gamma=0.8$, and $\beta=2.1)$. (b) Dynamics with decreased maximum fecundity $(A=10, \gamma=0.37$, and $\beta=2.1$.

\section{A stochastic model with beetle aggregation}

In this section, we modify the simplified beetle density model (22) to account for nonuniform density of beetles due to aggregation. Here, we simply propose the model, leaving its analysis and application to beetle outbreaks for future work.

The simplified beetle density model (22) assumes that, for a given vigor level $v$, the density of beetles (number per unit area of bark) at time $t$ is given precisely by $b_{t}(v)$. Whether a local outbreak is successful depends on whether $b_{t}(v)$ exceeds the outbreak threshold (Fig. 1), yielding a value of $\chi$ near one. In reality, the density of beetles in a given unit of tree bark will vary, depending on whether the unit of tree bark contains a local aggregation of beetles. Even when average beetle levels are low, outbreaks of beetles can succeed locally if aggregations of beetles drive the local level of $\chi$ close to one. 


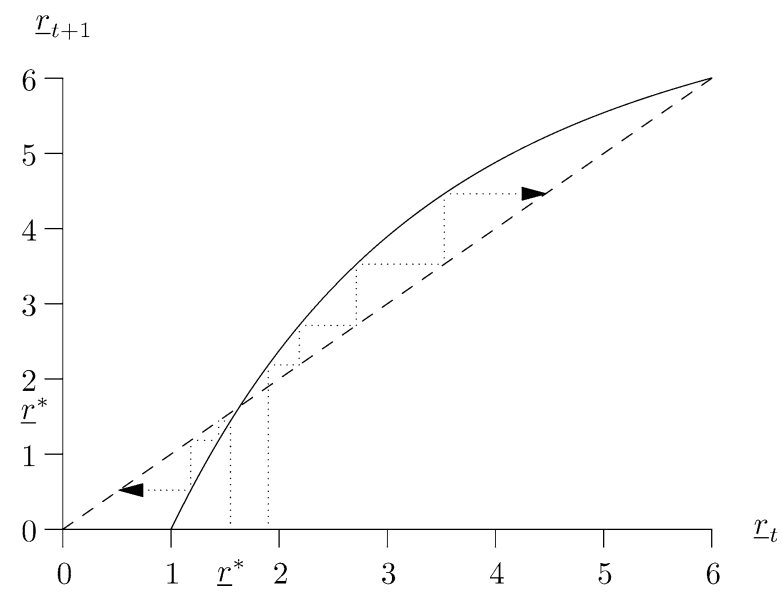

Fig. 7 Cobwebbing the nonlinear threshold model (39). $r_{t}$ denotes the ratio of beetle densities from one time step to the next. The initial value is $r_{0}=\tilde{b}_{0}=N_{0}(1-\exp (-c)) / c$ where $N_{0}$ is the total number of beetles and $c$ is vigor preference parameter. If $r_{t}$ drops below one, the population dies out.

One approach to modeling insect aggregation is to simulate its spatial structure explicitly using partial differential equations or related models (Powell et al., 2000). Another approach describes variation in the insect densities using the ideas of random variables: noninteracting insects are Poisson distributed on their hosts, while aggregating insects are overdispersed (variance exceeds the mean). This second approach has been widely used in the context of host-parasitoid dynamics (Hassell, 1978) where the host is an insect larvae, and the aggregating insect is the parasitoid fly or wasp. We follow this second approach for modeling mountain pine beetle aggregation. In our case, the host is in a given unit area of tree bark, and the aggregating insect is the pine beetle.

We describe density of beetles on bark with vigor $v$ as a random variable $B_{t}(v)$ with expected value $b_{t}(v)$. Recall that the nondimensionalization (19) calculates the units for $b_{t}(v)$ as density relative to the low-vigor threshold density $\beta_{0}$. In other words, a value of $B_{t}(v)$ exceeding one is sufficient to kill a tree, providing $v$ is very small. The random variable $B_{t}(v)$ can be translated into the number of beetles on a tree host of area $A_{T}$. This is through multiplication by the dimensionless scaling factor $\beta_{0} A_{T}$ to give $B_{t}(v) \beta_{0} A_{T}$. If beetles act independently of one another, the number of beetles on a tree of area $A_{T}$ should be Poisson distributed, with mean $b_{t}(v) \beta_{0} A_{T}$, and a corresponding variance also equal to $b_{t}(v) \beta_{0} A_{T}$. However, beetle aggregation will cause the beetles to overdisperse so that the variance exceeds the mean. This additional variation in beetle levels will translate into increased variation in outbreak success for any given value of $b_{t}(v)$. Translating back to the dimensionless beetle density, a Poisson distributed beetle population will have a mean of $b_{t}(v)$ and corresponding variance equal to $\left(\beta_{0} A_{T}\right)^{-1}$, and an overdispersed beetle population will have variance exceeding $\left(\beta_{0} A_{T}\right)^{-1}$.

Classical approaches to insect aggregation use a negative binomial random variable for the number of overdispersed insects on a host (Hassell, 1978). While this approach would be possible for mountain pine beetle, we adopt a more flexible approach, using a continuous random variable $B_{t}(v)$ with probability density function $g\left(b ; b_{t}(v), \theta\right)$ that has mean $b_{t}(v)$ and dispersion parameter $\theta$. 
Equation (22) is interpreted as the equation for the expected density of beetles, and is rewritten as

$$
b_{t+1}(v)=\tilde{k}(v) \frac{\left(\int_{0}^{1} \phi_{t}(v) \int_{0}^{\infty} g\left(b ; b_{t}(v), \theta\right) \chi(b, v) b A e^{-\gamma b} d b d v\right)}{\int_{0}^{1} \tilde{k}(v) \phi_{t}(v)\left(1-\int_{0}^{\infty} g\left(b ; b_{t}(v), \theta\right) \chi(b, v) d b\right) d v},
$$

As the threshold function $\chi$ becomes steep $(\alpha \rightarrow \infty)$ is it

$$
b_{t+1}(v)=\tilde{k}(v) \frac{\left(\int_{0}^{1} \phi_{t}(v) \int_{\exp \left(\beta_{1} v\right)}^{\infty} g\left(b ; b_{t}(v), \theta\right) b A e^{-\gamma b} d b d v\right)}{\int_{0}^{1} \tilde{k}(v) \phi_{t}(v) G\left(\exp \left(\beta_{1} v\right) ; b_{t}(v), \theta\right) d v},
$$

where $G\left(b ; b_{t}(v), \theta\right)$ is the cumulative density function for $b$. The corresponding equation for the distribution of bark area of live hosts as a function of host vigor (17) becomes

$$
\phi_{t+1}(v)=\phi_{t}(v) G\left(\exp \left(\beta_{1} v\right) ; b_{t}(v), \theta\right) .
$$

One possible form for $g\left(b ; b_{t}(v), \theta\right)$, consistent with observations of beetle densities of zero in uninfested trees and approximately $60 \mathrm{~m}^{-2}$ in infested trees, would be bimodal, with peaks at $b=0$ and $b=b_{m} \approx 3$ (in nondimensional variables). While this appears to be a fruitful avenue for development, we leave it for future research.

In some cases, the integrands in Eq. (42) can be simplified. For example, if the probability density function $g$ is chosen as the gamma distribution with mean $b_{t}(v)$ and variance $b_{t}^{2}(v) / \theta$

$$
g\left(b ; b_{t}(v), \theta\right)=\frac{b^{\theta-1} e^{-\theta b / b_{t}(v)}}{\Gamma(\theta)\left(b_{t}(v) / \theta\right)^{\theta}},
$$

with corresponding cumulative density function

$$
\operatorname{Pr}\left(B_{t}<b\right)=G\left(b ; b_{t}(v), \theta\right)=1-\frac{\Gamma\left(\theta, b \theta / b_{t}(v)\right)}{\Gamma(\theta)},
$$

where $\Gamma(\cdot)$ is the gamma function and $\Gamma(\cdot, \cdot)$ is the incomplete gamma function (Fig. 8), then in the limit $\theta \rightarrow \infty, g\left(b ; \theta, b_{t}\right) \rightarrow \delta\left(b-b_{t}\right)$ and $G\left(b ; \theta, b_{t}\right) \rightarrow H\left(b-b_{t}\right)$ and model (22) is regained (Fig. 8). Using the cumulative density function (45), the numerator of (42) can be rewritten as

$$
A \int_{0}^{1} \phi_{t}(v) h\left(b_{t}(v)\right) d v
$$

where

$$
h\left(b_{t}(v)\right)=b_{t}(v)\left(\frac{\theta}{\theta+\gamma b_{t}(v)}\right)^{\theta+1}\left(1-G\left(e^{\beta_{1} v}, \frac{(\theta+1) b_{t}(v)}{\theta+\gamma b_{t}(v)}, \theta+1\right)\right) .
$$

The variance of the gamma distribution is given as $b_{t}^{2} / \theta$ and hence the population is overdispersed when $b_{t}^{2} / \theta>\left(\beta_{0} A_{T}\right)^{-1}$, or equivalently $\theta<b_{t}^{2}(v) \beta_{0} A_{T}$. For a beetle population near attack threshold density $b_{t}(\nu) \approx 1$, and the inequality for an overdispersed population is given approximately by $\theta<\beta_{0} A_{T} \approx 281$ (Table 2 ). 

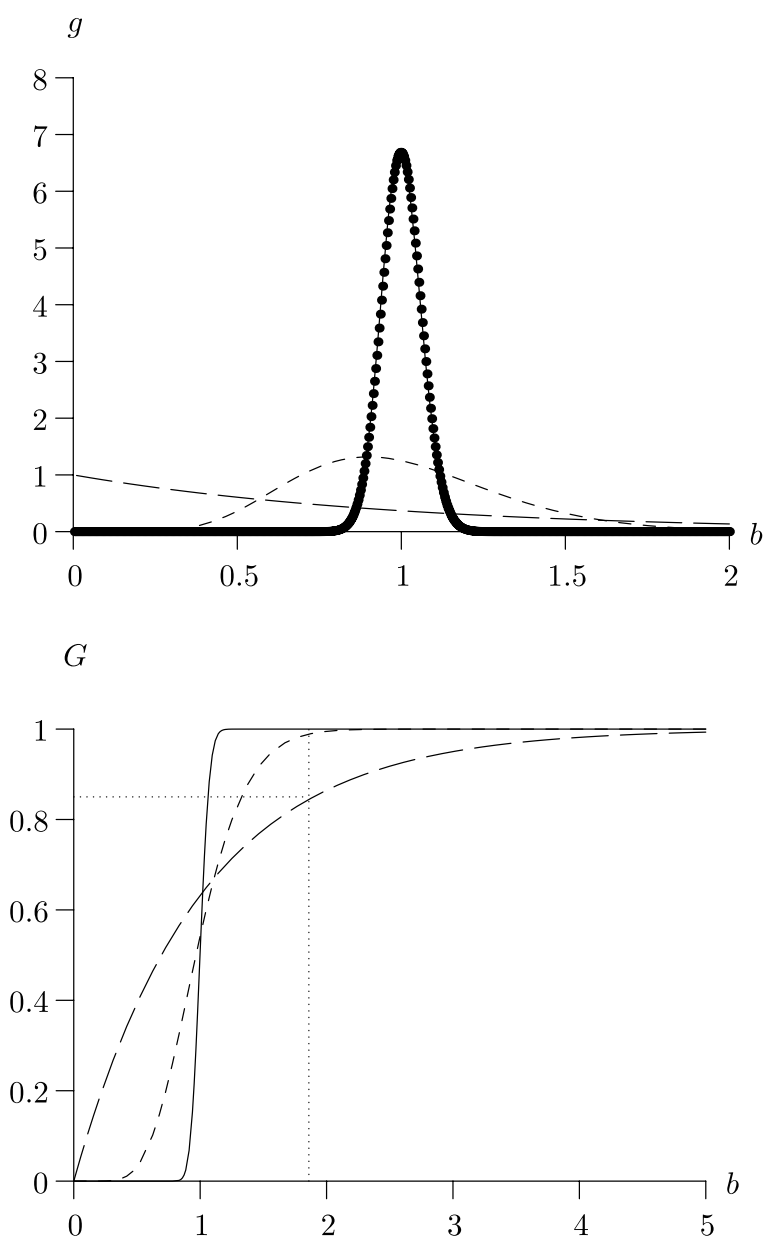

Fig. 8 The gamma function can be used to describe beetle aggregation. The probability density function (44) (top panel) and cumulative density function (45) (bottom panel) for a gamma distribution of beetles are shown for mean beetle density $b_{t}(v)=1$. Dispersion parameters are $\theta=1$ (exponential, long dashed), $\theta=10$ (dashed) and $\theta=281$ (solid). Lower $\theta$ levels indicate higher aggregation levels. At $\theta \rightarrow \infty$ the beetle density is given by its mean, and the model becomes deterministic. The dots indicate the Poisson distribution rescaled to correspond with the $\theta=281$ case. This is approximated closely by the solid line arising from the gamma distribution. To observe the effect of aggregation, observe that for $\beta_{1}=2.1$ and $v=0.25$ the threshold for successful attack, $\exp \left(\beta_{1} v\right)=1.86$ is exceeded by about $15 \%$ of the beetles with dispersion parameter $\theta=1$, but almost none of beetles with larger $\theta$.

Figure 8 shows the gamma distribution for various $\theta$ values, and demonstrates that this distribution (solid line) also closely approximates the nonaggregating Poisson model (dots) for the appropriate value of $\theta(\theta=281)$. For this value of $\theta$, the probability density function approaches a delta function and the cumulative density function approaches a step function, indicating that the behavior of this nonaggregating model will be close to that of the deterministic model (22). 
The gamma distribution allows for the possibility that, even under heavy beetle attack, the most common observation (mode) would be trees with no attacks (Fig. 8, long-dashed line). This arises in the limit $\theta \rightarrow 1$ as (44) becomes an exponential distribution $g$. The lower panel of Fig. 8 illustrates how, when the average beetle density lies below that threshold level for attack success, higher levels of aggregation (lower $\theta$ ) mean that a larger fraction of attacks are successful.

Even with the simplification of a gamma distribution for $g$, Eqs. (42)-(43) must be solved numerically using quadrature. The numerical solution of these equations, for varying values of beetle aggregation $(\theta)$, is shown in Fig. 9. The $\theta$ values for beetle aggregation are as illustrated in Fig. 8. The top row of Fig. 9 shows no aggregation $(\theta=281)$, while the second and third rows of the figure show increasing levels of aggregation $(\theta=10$ and $\theta=1$, respectively). (The corresponding probability density and cumulative density functions for the aggregation functions are shown in Fig. 8.) All other parameters in the model are chosen to be identical to the top panels of Fig. 5 (left column) and Fig. 6 (right column). Note that the top panel of the left column of Fig. 9 (stochastic model with linear dynamics and no aggregation) is similar to the top panel of Fig. 5 (deterministic model with linear dynamics and no aggregation), and that the top panel of Fig. 9 (stochastic model with nonlinear dynamics and no aggregation) is similar to the top panel of Fig. 6 (deterministic model with nonlinear dynamics and no aggregation).

As with the previous figures, the black dashed, solid white, and white dashed lines show threshold beetle abundances, as calculated for the deterministic models. They are simply reproduced in this figure for reference. Lines in the left column are identical to those in the top panel of Fig. 5. The black dashed line indicates the minimum beetle abundance required to kill the weakest hosts in the deterministic model with no aggregation; the solid white line indicates the minimum beetle abundance guaranteed to eventually kill the entire stand in the deterministic model with no aggregation; and white dashed line indicates the minimum beetle abundance guaranteed to kill the stand in a single year in the deterministic model with no aggregation. In a similar manner, lines in the right column are identical to those in the top panel of Fig. 6.

Note that the grey area in each panel increases with increasing aggregation. This can be seen as one moves from the top row to the bottom row in either column. This means that increased aggregation makes it more likely that a stand will be successfully attacked (white areas are replaced by grey), but also makes it more likely that a fraction of the stand will escape being killed (black areas are replaced by grey). We interpret this result as follows: at low beetle abundance, aggregation helps ensure that the attack threshold is exceeded in some trees, and hence that some individuals in a stand become infected. However, at high abundance, aggregation means that certain trees fall below the attack threshold as the beetles move to other trees, and hence the entire stand is not infected at levels above threshold.

\section{Discussion}

Previous productivity curve models have modeled beetle production in an entire stand. This does not easily account for the changing vigor structure as the beetle infestation progressed. In this paper, we have modeled and analyzed a vigor-structured model for beetle dynamics within a stand. This model explicitly tracks the changing vigor structure in the 

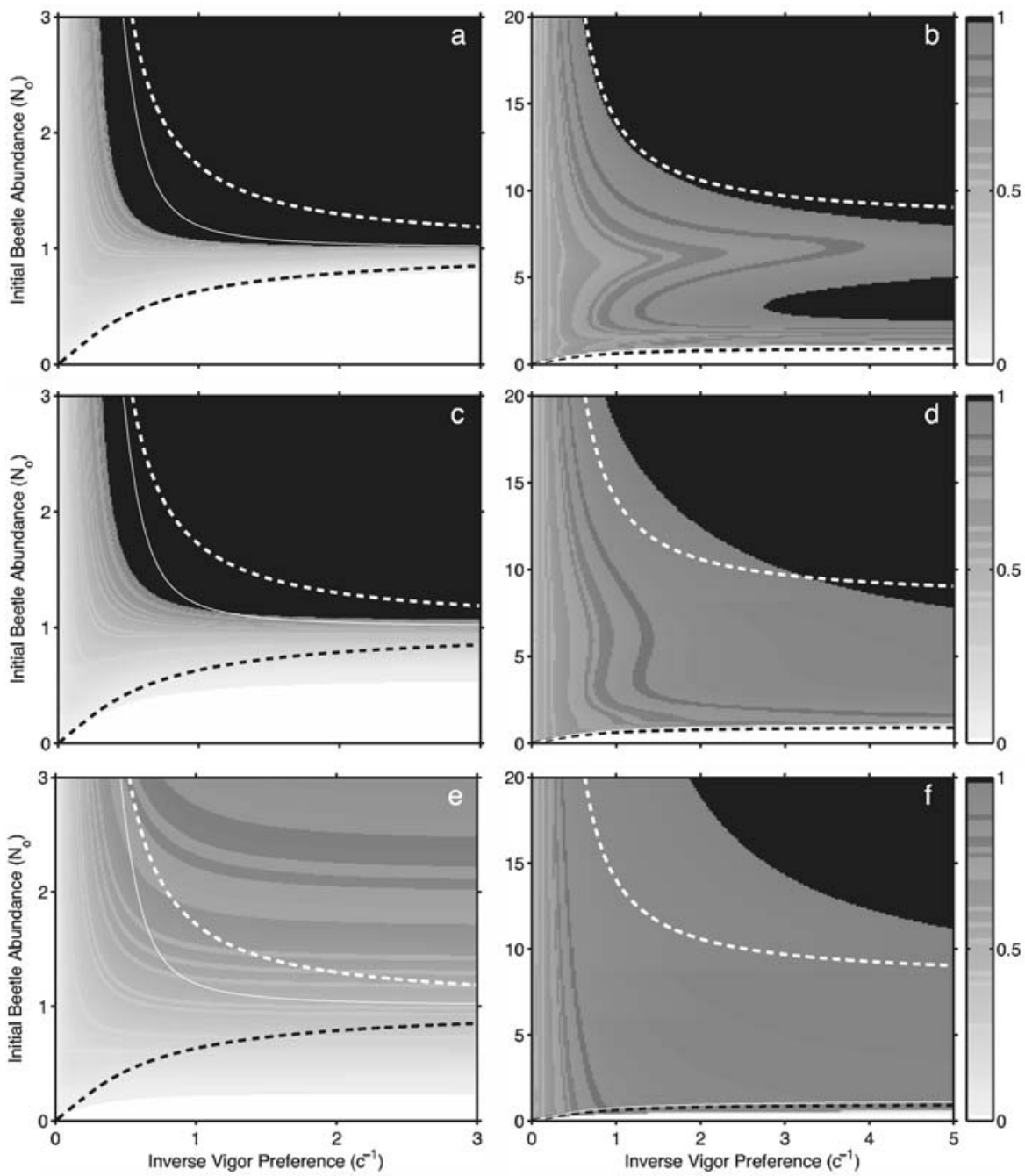

Fig. 9 Forest damage as a function of initial beetle density predicted by the stochastic threshold model. Dynamics parameters in the left column are $\alpha=2, \gamma=0.8$ and $\beta=0$, and dynamics parameters in the right column are $\alpha=33, \gamma=0.8$ and $\beta=2.1$. Aggregation parameters are $\theta=281$ in the first row, and $\theta=10$ and 1 in the second and third rows. Note the grey area in each panel increases with increasing aggregation, as one moves from the top row to the bottom row.

stand. All model parameters, other than vigor preference $c$ and dispersal survival $S$, were determined by fitting model components to empirical data (Table 2). The closest existing model to the one developed here is the computer simulation model of Raffa and Berryman (1986). However, that model did not provide an explicit mathematical formulation, such as the one given here. We hope that our explicit mathematical formulation provides a foundation for basis further insight and analysis by other researchers. 
The added detail needed to track vigor structure has generated mathematical complexity; the models (1)-(2), (7) and (13) are a system of nonstandard nonlinear integrodifference equations. However, the assumption of an abrupt threshold response $(\alpha \rightarrow \infty)$ allowed for model simplification, given by reduction to a delayed discrete-time dynamical system for beetle population levels. Analysis of this simplified model allowed for predictions of model outcomes (fraction of stand killed by beetles) as a function of the two unknown quantities: initial number of beetles introduced to the stand $N_{0}$ (variable) and vigor preference $c$ (see Figs. 5 and 6).

Our assumption that beetles respond precisely to the low tree vigor is an simplification, as they cue on a large number of chemical signals of which tree kairomones are just one. In addition, the beetles may cue on larger tree size, which often corresponds with low tree vigor. As mentioned in Section 2.2 our simplification can be considered a "best-case" establishment scenario, where by choosing low-vigor trees, beetles can establish most easily. In our example, we consider a vigor distribution that is initially uniform. Although this is a useful starting point, it likely that the initial vigor distribution is structured, possibly with peaks at low and high vigor. It is intriguing to consider whether such structure could give rise to meta-stable dynamics, where beetles slowly build up populations in a small number low-vigor hosts before breaking out and attacking the high vigor hosts (cf. Safranyik and Carroll, 2006).

A model extension to account for beetle aggregation used ideas from the models for host-parasitoid dynamics with aggregating parasitoids (Hassell, 1978). This model allows researchers to use empirically derived distributions of host selection and aggregation to describe complex spatial processes. This stochastic formulation is quite general, but adds an additional level of model complexity. One possible approach would be to choose the bimodal aggregation function, described in Section 4, right after Eq. (43).

Although likely less realistic for mountain pine beetle than the bimodal distribution, the gamma distribution provides a flexible, but relatively tractable distribution for aggregating beetle numbers on the host trees. Using this, the effect of aggregation on outbreak dynamics can be analyzed numerically, as shown in Fig. 9. Here, the numerical results show that the effect of aggregation is to increase the likelihood of successful attack of a stand, but to decrease the likelihood that the entire stand is killed. Further work is needed to estimate the appropriate aggregation parameter for the mountain pine beetle.

The work of Nelson et al. (2008) asks why current risk models fail, concluding that the primary reason is because beetle density has been removed as a hazard index in current risk models. As shown graphically in Figs. 5, 6, and 9, our model has provided a framework where beetle density is a key factor determining forest damage. Our model has underscored the role of initial population levels $N_{0}$, as well as selective preference of beetles for low vigor classes $c$, and the nonlinear attack threshold $\beta_{1}$ in determining the attack success and, when successful, attack outcomes (entire forest killed versus a fraction).

Our goal has been to develop a strategic model which can be used to relate risk of forest damage to known quantities. There are additional processes, not included in our model, that govern dynamics in natural stands. These include competition with other bark beetles (Safranyik and Carroll, 2006), variable redistribution survivorship $S$ (Burnell, 1997), evidence that host selection can change with beetle density (Wallin and Raffa, 2004) and temperature-dependent beetle phenology (Bentz et al., 1991). While strategic models that leave out too many ecological factors may be unable to predict risk may be unable to 
predict risk (Nelson et al., 2008), we believe that the next step is to validate the vigorstructured model against mountain pine beetle infestation data. Based on the outcome of this, it may be necessary to develop the model further. This will be the subject of further work.

\section{Acknowledgements}

This study was funded by Natural Resources Canada-Canadian Forest Service under the Mountain Pine Beetle Initiative. Publication does not necessarily signify that the contents of this report reflect the views or policies of Natural Resources Canada-Canadian Forest Service. The first author was also supported by an NSERC Discovery grant and Canada Research Chair. The second author was also supported by NSERC and Alberta Ingenuity Fund postdoctoral fellowships. The third author was supported by a Killam postdoctoral fellowship. Thanks to three anonymous reviewers and to the Lewis Lab for helpful suggestions that have improved this paper. Thanks also to members of the TRIA Project (Mountain Pine Beetle System Genomics).

\section{Appendix A: Notes for converting axes in Fig. 1(b)}

The axes used is the original manuscript by Mulock and Christiansen (1986) were $Y=\frac{A 20}{D}$, where $A$ is the number of successful attacks on the entire host and $D$ is the diameter at breast height of the tree, and $X=\frac{B}{S}$, where $B$ is the yearly increase in a tree's basal area, and $S$ is the sapwood area. We seek to transform these data onto new axes of $Y^{*}=A^{*}$, where $A^{*}$ is the number of attacks per meter square of bark area, and $X^{*}=\frac{M}{L}$, where $M$ is the yearly mass gain of the tree and $L$ is the leaf area of the tree. To convert each data point, we would need the height, sapwood area, and diameter of each tree. Since this information is unavailable, we instead transform the data using the average value of these quantities for the stand as follows: $Y^{*}=\frac{Y 100}{20 H \pi}$, where $H$ is the maximum height of the attacks (Mulock and Christiansen, 1986). The factor of 100 converts the diameter in meters to bark area in meters. Host vigor is converted as $X^{*}=\frac{X S^{*} H^{*} \delta}{100 L^{*}}$, where $S^{*}$ is the average sapwood area in a stand, $\delta$ is the density of wood, $H^{*}$ is the average tree diameter in centimeters, and $L^{*}$ is the allometric relationship relating sapwood area to leaf area in Norway Spruce (Stancioiu and O’Hara, 2006) (Table A.1).

Table A.1 Parameters for converting the axes in Fig. 1(b)

\begin{tabular}{lll}
\hline Parameter & Estimate & Description \\
\hline$H$ & $18.5 \mathrm{~m}$ & Maximum attack height \\
$H^{*}$ & $1850 \mathrm{~cm}$ & Maximum attack height \\
$S^{*}$ & $25 \mathrm{~cm}$ & Average sapwood area \\
$\delta$ & $.322 \mathrm{~g} \mathrm{~cm}^{-3}$ & Wood density \\
$L^{*}$ & 10 & Relationship between sapwood area and leaf area \\
\hline
\end{tabular}




\section{Appendix B: Methods for estimating parameters}

Parameter estimates were derived from two studies on mountain pine beetles. Beetle parameters were estimated from data in Raffa and Berryman (1983), and host parameters were estimated from data in Waring and Pitman (1985).

\section{B.1 Beetle parameters $(A, \gamma)$}

Raffa and Berryman (1983) report observations of the number of pupae produced per attack as a function of attack density. If we assume that the mortality from pupae to adult is small, then these data provide the necessary information to estimate $\mathrm{A}$ and $\gamma$ for that particular stand, in the year that the study was undertaken. The data were digitized and the parameters estimated by fitting the following nonlinear function $p(b(a, v), v)=$ $A \exp (-\gamma b(a, v))$. The minimization was done using the NLS library in the R statistical environment.

\section{B.2 Host parameters $\left(\alpha, \beta_{0}, \beta_{1}, v_{m}\right)$}

Waring and Pitman (1985) present a data set of host mortality as a function of attacking beetle density and host vigor for mountain pine beetles in a lodgepole pine stand. We use this data to obtain estimates for $\alpha, \beta_{0}$, and $\beta_{1}$. A small number of the data were for 'strip-attacks' where only a portion of the tree is killed. Since the extent of the damage is not reported, and since they only represent a small proportion of the data set, we exclude them from the analysis. The expected probability of host mortality $\left(\pi_{i}\right)$ is given by $\pi_{i}=$ $\left(1+\exp \left(-\alpha\left(y_{i}-\beta_{0} \exp \left(\beta_{1} x_{i}\right)\right)\right)\right)^{-1}$ where $x_{i}$ is the vigor of host $i$, and $y_{i}$ is the density of attacking beetles. Since the response variable is binary (host is either dead or alive), the parameters were estimated by minimizing the log-likelihood objective function $(L)$ for binary data $L=-\sum_{i=1}^{n}\left(z_{i} \log \left(\frac{\pi_{i}}{1-\pi_{i}}\right)+\log \left(1-\pi_{i}\right)\right)$ where $z_{i}$ is the observed host outcome (0 or 1$)$. The minimization was done using the optim library in the $\mathrm{R}$ statistical environment. To avoid numerical errors, the predicted probability of host mortality was bound between 0.0001 and 0.999 .

The maximum vigor $\left(v_{m}\right)$ was taken as the maximum vigor observed in the stand.

\section{References}

Bentz, B.J., Logan, J.A., Amman, G.D., 1991. Temperature-dependent development of mountain pinebeetle (coleoptera, scolytidae) and simulation of its phenology. Can. Entomol. 123, 1083-1094.

Berryman, A.A., 1979. Dynamics of bark beetle populations: Analysis of dispersal and redistribution. Bull. Lociete Entomol. Suisse 52, 227-234.

Berryman, A.A., Stenseth, N.C., Wollkind, D.J., 1984. Metastability of forest ecosystems infested by bark beetles. Res. Popul. Ecol. 26, 13-29.

Burnell, D.G., 1997. A dispersal-aggregation model for mountain pine beetle in lodgepole pine stands. Res. Popul. Ecol. 19, 99-106.

Caswell, H., Nisbet, R.M., de Roos, A.M., Tuljapurkar, S., 1997. Structured-population models: many methods, a few basic concepts. In: Tuljapurkar, S., Caswell, H. (Eds.), Structured-population Models in Marine, Terrestrial, and Freshwater Systems. Chapman and Hall, London

Cole, W.E., McGregor, M.D., 1983. Estimating the rate and amount of tree loss from mountain pine beetle infestations. Technical Report Res. Pap. INT-318, U.S. Department of Agriculture, Forest Service, Intermountain Forest and Range Experiment Station 
Gurney, W.S.C., Nisbet, R.M., 1998. Ecological Dynamics. Oxford University Press, Oxford.

Hassell, M.P., 1978. The Dynamics of Arthropod Predator-Prey Systems. Princeton Monographs in Population Biology. Princeton University Press, Princeton.

He, F., 2006. Observations of mountain pine beetle infestations of lodgepole pine stands in southern British Columbia. Unpublished

Logan, J.A., Powell, J.A., 2001. Ghost forests, global warming, and the mountain pine beetle. Am. Entomol. 47, 160-173.

Logan, J.A., White, P., Bentz, B.J., Powell, J.A., 1998. Model analysis of spatial patterns in mountain pine beetle outbreaks. Theor. Popul. Biol. 53, 236-255.

Logan, J.A., Regniere, R., Powell, J.A., 2003. Assessing the impacts of global warming on forest pest dynamics. Front. Ecol. Environ. 1, 130-137.

Moeck, H.A., Simmons, C.S., 1991. Primary attraction of mountain pine beetle Dendroctonous ponderosae Hopk. (Coloeptera: Scolytidae), to bolts of lodgepole pine. Can. Entomol. 123, 299-304.

Mulock, P., Christiansen, E., 1986. The threshold of successful attack by Ips Typographus on Picea abies: a field experiment. For. Ecol. Manag. 14, 125-132.

Nelson, W.A., Potapov, A., Lewis, M.A., Hundsdorfer, A.E., He, F., 2008. Balancing ecological complexity in predictive models: a reassessment of risk models in the mountain pine beetle system. J. Appl. Ecol. 45, 248-257.

Powell, J.A., Logan, J.A., Bentz, B.J., 1996. Local projections for a global model for mountain pine beetle attacks. J. Theor. Biol. 179, 243-260.

Powell, J.A., Kennedy, B., White, P., Bentz, B.J., Logan, J.A., Roberts, D., 2000. Mathematical elements of attack risk analysis for mountain pine beetles. J. Theor. Biol. 204, 601-620.

Raffa, K.F., Berryman, A.A., 1983. The role of host plant-resistance in the colonization behavior and ecology of bark beetles Coleoptera, Scolytidae. Ecol. Monogr. 53, 27-49.

Raffa, K.F., Berryman, A.A., 1986. A mechanistic computer-model of mountain pine-beetle populations interacting with lodgepole pine stands and its implications for forest managers. For. Sci. 32, 789-805.

Safranyik, L., Carroll, A., 2006. The biology and epidemiology of the mountain pine beetle in lodgepole pine forests. In L. Safranyik, B. Wilson (Eds.) The Mountain Pine Beetle: A Synthesis of Its Biology and Management in Lodgepole Pine, Natural Resources Canada, Canadian Forest Service.

Stancioiu, P.T., O'Hara, K.L., 2006. Leaf area and growth efficiency of regeneration in mixed species, multiaged forests of the Romanian Carpathians. For. Ecol. Manag. 222, 55-66.

Wallin, K.F., Raffa, K.F., 2004. Feedback between individual host selection behavior and population dynamics in an eruptive herbivore. Ecol. Monogr. 74, 101-116.

Waring, R.H., Pitman, G.B., 1985. Modifying lodgepole pine stands to change susceptibility to mountain pine beetle attack. Ecology 66, 889-897.

White, P., Powell, J., 1997. Phase transition from environmental to dynamic determinism in mountain pine beetle attack. Bull. Math. Biol. 59, 609-643.

White, P., Powell, J., 1998. Spatial invasion of pine beetles into lodgepole forests: a numerical approach. SIAM J. Sci. Comput. 20, 164-184. 